\title{
Doença de Chagas aguda no estado do Maranhão, Brasil: uma comparacã̃o entre os baneos de dados do SINAN e da FUNASA
}

\author{
Acute Chagas Disease in the state of Maranhao, Brazil: \\ a comparison between the databases SINAN and FUNASA
}

\author{
José Ribamar Amorim Rodrigues \\ Jorge Luís Ramos da Silva-Júnior' \\ Aleyone de Oliveira Paredes ${ }^{2}$ \\ Aramys Silva Reis ${ }^{3}$ \\ Luegya Alves de Garvalho Silva ${ }^{4}$
}

\section{Resumo}

A doença de Chagas é uma parasitose endêmica e com alta prevalência na América Latina. O objetivo desse trabalho foi comparar os dados de notificação da doença no SINAN (Sistema de Informação de Agravos de Notificação) e na FUNASA (Fundação Nacional de Saúde) e avaliar o perfil da doença no Estado do Maranhão. Foram utilizados os dados da Secretaria de Saúde do Estado e da FUNASA-MA de 2007 a 2011. A análise resultou em divergências entre as duas fontes de informações quanto ao número de casos por ano, cidades afetadas e, principalmente, em relação ao modo de transmissão. Os indivíduos mais atingidos foram os da fase adulta, pertencentes ao sexo masculino e moradores da zona rural. Além disso, a doença apresentou uma baixa mortalidade. A falta de treinamento dos técnicos responsáveis pela coleta e abastecimento dos dados e até mesmo o diagnóstico incorreto são fatores que podem estar envolvidos nas divergências entre os dois bancos de dados. Logo, é necessária a busca de mecanismos que busquem melhorar a vigilância epidemiológica e obtenção de dados mais confiáveis da Doença de Chagas.

\section{Abstraet}

Chagas disease is an endemic parasitosis highly prevalent in Latin America. The aim of this study was to compare the notification disease data in SINAN and FUNASA and evaluate the profile of the disease in the state of Maranhao. This study analyzed data from the Secretary of State Health and FUNASA-MA within the last five years. Analysis showed discrepancies between the two databases regarding the number of cases per year, affected cities, and especially related to the mode of transmission. People in adulthood, males and rural residents were the mainly infected. Moreover, the disease presented a low mortality. The lack of trained technicians responsible for collecting and supplying the data and even the correct diagnosis are factors that are involved in the differences between the two databases. Therefore, it is necessary the creation of mechanisms to improve epidemiological surveillance and obtaining more reliable data about Chagas Disease.
'Farmacêutico. Instituto Florence de Ensino Superior

${ }^{2}$ Farmacêutiea. Professora do Instituto Florence de Ensino Superior. Mestranda em Saúde Materno-Infantil [UFMA]. Universidade Federal do Maranhão

${ }^{3}$ Farmacêutieo, Mestre em Ciêneias da Saúde [uFMA]. Doutorando en Ciêneias [Parasitologia]. Universidade de São Paulo [USP].

${ }^{4}$ Farmacêutica. Professora Mestre do Instituto Florenee de Ensino Superior. Doutoranda em Biotecnologia [RENORBIO]. Universidade Federal do Maranhão [UFMA]

Para correspondência:

Luecya Alves de Carvalho da Silva

email: luecya_carvalho@hotmail.com 
Introdụão

A Doença de Chagas (DC) é uma parasitose de natureza endêmica na América Latina e Caribe que afeta aproximadamente 10 milhões de pessoas em todo o mundo1. Cerca de 300.000 novos casos são diagnosticados por ano, representando uma importante causa de mortalidade em adultos jovens ${ }^{2}$.

As formas habituais de transmissão da doença de Chagas são aquelas ligadas diretamente ao vetor, proveniente do contato do indivíduo com as fezes ou urina contaminadas com o protozoário Trypanosoma cruzi ${ }^{3}$. Entretanto, a transfusão de sangue contaminado, acidentes laboratoriais, transmissão congênita e transmissão por via oral também podem ocasionar o processo infeccioso ${ }^{4,5}$.

Até a década de 70, não haviam sido notificados nenhum caso de DC no Estado do Maranhão. Entretanto, em 1975 surgiram três casos provenientes da Ilha de São Luis e um da Baixada Maranhense, sendo que em todo o estado, a prevalência de infecção era de $0,1 \%{ }^{6}$.

Diversos levantamentos têm sido realizados, desde então, com o objetivo de caracterizar as condições sociodemográficas, espaço-temporal, faixa etária e manifestações clínicas desta doença no Maranhão. De acordo com o Ministério da Saúde, em 1996 foi realizado um levantamento entomológico, onde foram capturados 290.576 exemplares de triatomíneos no país. Do total de 201.156 encontrados na região Nordeste, 1.240 foram provenientes do estado do Maranhão, sendo que 35\% destes estavam infectados com T.cruzi ${ }^{7}$.

Posteriormente, Cutrim et al $(2010)^{7}$ avaliaram as características sociodemográficas e ambientais envolvidas na transmissão do protozoário no Estado do Maranhão. Este levantamento, realizado entre os anos de 1994 a 2008, mostrou que durante esse período, a infecção no estado é predominantemente vetorial, no ambiente silvestre e peridomiciliar.

Atualmente o Maranhão não é considerado um estado endêmico para a DC, entretanto, tem sido observado um crescimento expressivo no número de casos, embora nem todos sejam notificados adequadamente nos órgãos competentes. Atualmente, os órgãos responsáveis pelo registro de casos da doença são o Sistema de Informação de Agravos de Notificação (SINAN) e a Fundação Nacional de Saúde (FUNASA). Estes têm por objetivo facilitar a formulação e avaliação das políticas, planos e programas de saúde, subsidiando o processo de tomada de decisões, com vistas a contribuir para a melhoria da situação de saúde da população ${ }^{8}$. Porém, frequentemente, é comum verificar erros de notificação e até muitos casos de subnotificação ao sistema, o que prejudica as políticas de controle da doença.

A notificação adequada possui grande importância, pois através dela é possível traçar um perfil epidemiológico e, a partir daí, elaborar um planejamento para prevenção e tratamento eficaz da doença. Entretanto, para que isto ocorra os dados precisam ser notificados sem divergência e o diagnóstico da doença precisa ser feito quando a mesma ainda se encontra em estágio primário.

Nesse sentido, o presente estudo tem por objetivo realizar uma comparação entre os casos de Doença de Chagas Aguda (DCA) notificadas ao SINAN e à FUNASA no Estado do Maranhão entre 2007 e 2011, verificando a confiabilidade das notificações e o perfil da doença no Estado.

\section{Metodologia}

Trata-se de um estudo longitudinal, descritivo, retrospectivo e comparativo entre os bancos de dados do SINAN e da FUNASA, referentes às notificações de casos agudos de Doença de Chagas no período de 2007 a 2011, no Estado do Maranhão.

Os dados do SINAN foram coletados na Superintendência de Epidemiologia e Controle de Doenças (Secretaria de Estado da Saúde) no Departamento de Epidemiologia com o auxílio do softwareTabwim, programa que faz a tabulação dos dados do SINAN. Os dados da FUNASA foram obtidos através dos livros de registro de casos disponíveis na sede regional da fundação no município de São Luis, MA.

Para o estudo foram considerados os casos notificados ao SINAN e/ou à FUNASA como "Doença de Chagas Aguda" nos municípios do Estado do Maranhão entre janeiro de 2007 e dezembro de 2011. Para demonstrar a distribuição dos casos da doença no Estado, os municípios foram divididos segundo o Instituto Brasileiro de Geografia e Estatística - IBGE $^{9}$ em mesorregiões (Centro Maranhense, Leste Maranhense, Norte Maranhense, Oeste Maranhense e Sul Maranhense).

Como critérios de inclusão, os pacientes deveriam residir no Maranhão na data da manifestação da doença. Para o estudo foram consideradas as seguintes variáveis: sexo, zona de moradia e idade (faixa pediátrica - 0 a 11 anos; faixa juvenil - 12 a 18 anos; faixa adulta - 19 a 59 
anos; idosa - $\geq$ a 60 anos). Para o perfil da doença foram analisados: a incidência por ano, a forma de transmissão e evolução dos casos determinados.

Os dados foram analisados e tabulados, sendo sumarizados em frequência e porcentagem.

\section{Resultados}

Como mostrado na Tabela 1, durante o período de estudo houve um maior o número de casos de DCA registrados na FUNASA em relação ao SINAN. Enquanto o SINAN registrou 27 casos da doença, na FUNASA este número passou para 37. Essas divergências também foram verificadas quando feita a distribuição dos casos por anos (Tabela 1).
As mesorregiões Norte e Centro Maranhense reportaram o maior número de casos entre os anos de 2007 e 2011 no SINAN, porém, diferente da FUNASA, só foram registrados 8 casos $(29,62 \%)$ em ambas as regiões, sendo São Roberto, o município com o maior número de casos no Centro e Pinheiro na Mesorregião Norte. Contudo, a Mesorregião Oeste mostrou maior periodicidade nas infecções.

Além da divergência entre o número de casos reportados por região, existem diferenças quanto aos municípios afetados (Tabela 2). Durante o período de estudo, segundo a FUNASA, houve casos da doença nos municípios de São João do Sóter, Presidente Sarney, Rosário, São Luís,

\begin{tabular}{lcccccccc}
\hline & $\begin{array}{c}\text { População } \\
\text { MA }\end{array}$ & \multicolumn{3}{c}{ SINAN } & \multicolumn{3}{c}{ FUNASA } \\
\hline & & $\mathbf{N}$ & $\mathbf{\%}$ & Incidência & $\mathbf{N}$ & $\mathbf{\%}$ & Incidência \\
\hline 2007 & 6.118 .995 & 2 & 7,41 & 0,03 & 3 & 8,11 & 0,05 \\
2008 & 6.305 .539 & 6 & 22,22 & 0,10 & 5 & 13,51 & 0,08 \\
2009 & 6.367 .111 & 4 & 14,81 & 0,06 & 2 & 5,41 & 0,03 \\
2010 & 6.574 .789 & 0 & 0 & 0,00 & 2 & 5,41 & 0,03 \\
2011 & 6.645 .761 & 15 & 55,56 & 0,23 & 25 & 67,57 & 0,38 \\
TOTAL & & $\mathbf{2 7}$ & $\mathbf{1 0 0 , 0}$ & & 37 & $\mathbf{1 0 0 , 0}$ & \\
\hline
\end{tabular}

Fonte: Pesquisa Direta

Tabela 1. Distribuição dos casos de Doença de Chagas Aguda no Estado do Maranhão e registrados no banco de dados do SINAN e da FUNASA de acordo com o ano de notificação.

Em ambos os bancos de dados, o ano com maior número de notificações foi 2011, porém com valores discordantes. Nesse ano foram registrados 15 $(55,56 \%)$ casos da doença no SINAN e $25(67,56 \%)$ casos na FUNASA, com incidências de 0,21 e 0,38 casos por 100 mil habitantes, respectivamente. Em 2010, segundo o SINAN, não foi notificado nenhum caso de DCA no estado. A FUNASA, por sua vez, registrou 2 casos. Por outro lado, em 2009 foram registrados 4 casos $(14,82 \%)$ no SINAN, ao passo que na FUNASA foram apenas 2 casos $(5,41 \%)$.

Quanto a distribuição da doença entre as regiões do estado (Tabela 2), verificou-se que, de acordo com o banco de dados da FUNASA, a mesorregião Norte teve o maior número de registros da doença durante o período estudado, 16 casos $(43,24 \%)$, distribuídos entre seis municípios. O município de Pinheiro respondeu pelo maior número de casos da infecção, 9 casos $(56,25 \%)$, sendo todos concentrados no ano de 2011.
Lago da Pedra e Santa Luzia, sendo que não foram reportados ao SINAN. Contudo, alguns casos apontados pelo SINAN como, por exemplo, municípios de Coelho Neto, Chapadinha, São Vicente Ferrer, Tutóia e Porto Franco, não foram notificados pela FUNASA.

Em relação ao perfil dos pacientes, também foi possível identificar diferenças entre os dois bancos de dados (Tabela 3). Enquanto 59,26\% dos casos notificados no SINAN eram do sexo masculino e $40,74 \%$ do sexo feminino, na FUNASA o percentual de pacientes do sexo masculino afetados pela doença era maior $(62,16 \%)$. Nessa situação os casos abrangendo o sexo feminino foi $37,84 \%$ do total. Apesar dessas diferenças, em ambos os sistema o sexo masculino sempre foi o mais afetado.

Diferenças entre os dois bancos de dados também foram observadas quanto à faixa etária. Em ambos os sistemas a faixa etária mais afetada 


\begin{tabular}{|c|c|c|c|c|c|c|c|c|c|c|c|c|}
\hline & \multicolumn{2}{|c|}{2007} & \multicolumn{2}{|c|}{2008} & \multicolumn{2}{|c|}{2009} & \multicolumn{2}{|c|}{2010} & \multicolumn{2}{|c|}{2011} & \multicolumn{2}{|c|}{ TOTAL } \\
\hline & SINAN & FUNASA & SINAN & FUNASA & SINAN & FUNASA & SINAN & FUNASA & SINAN & FUNASA & SINAN & FUNASA \\
\hline $\begin{array}{c}\text { Centro } \\
\text { Maranhense }\end{array}$ & - & -- & -- & -- & -- & -- & -- & -- & 08 & 08 & 08 & 08 \\
\hline $\begin{array}{l}\text { Barra do } \\
\text { Corda }\end{array}$ & -- & -- & -- & -- & -- & -- & -- & -- & 03 & 03 & 03 & 03 \\
\hline São Roberto & -- & -- & -- & -- & -- & -- & -- & -- & 05 & 05 & 05 & 05 \\
\hline $\begin{array}{c}\text { Leste } \\
\text { Maranhense }\end{array}$ & -- & 01 & -- & -- & 01 & -- & -- & -- & 03 & 01 & 04 & 02 \\
\hline Caxias & -- & -- & -- & -- & -- & -- & -- & -- & 02 & 01 & 02 & 01 \\
\hline Coelho Neto & -- & -- & -- & -- & 01 & -- & -- & -- & -- & -- & 01 & -- \\
\hline Chapadinha & -- & -- & -- & -- & -- & -- & -- & - & 01 & -- & 01 & -- \\
\hline $\begin{array}{l}\text { S. João do } \\
\text { Soter }\end{array}$ & -- & 01 & -- & -- & -- & -- & -- & -- & -- & -- & -- & 01 \\
\hline $\begin{array}{c}\text { Norte } \\
\text { Maranhense }\end{array}$ & 01 & -- & 02 & 03 & 02 & 01 & -- & -- & 03 & 12 & 08 & 16 \\
\hline Axixá & 01 & -- & 01 & 01 & -- & -- & -- & -- & -- & -- & 02 & 01 \\
\hline Icatu & -- & -- & 01 & 01 & -- & -- & -- & -- & -- & -- & 01 & 01 \\
\hline Pinheiro & -- & -- & -- & -- & -- & -- & -- & -- & 03 & 09 & 03 & 09 \\
\hline Pres. Sarney & -- & -- & -- & 01 & -- & -- & -- & -- & -- & -- & -- & 01 \\
\hline Rosário & -- & -- & -- & -- & -- & 01 & -- & -- & -- & 01 & -- & 02 \\
\hline $\begin{array}{l}\text { S. Vicente } \\
\text { Ferrer }\end{array}$ & -- & -- & -- & -- & 01 & -- & -- & -- & -- & -- & 01 & -- \\
\hline São Luís & -- & -- & -- & -- & -- & -- & -- & -- & -- & 02 & -- & 02 \\
\hline Tutoia & -- & -- & -- & -- & 01 & -- & -- & -- & -- & -- & 01 & -- \\
\hline $\begin{array}{c}\text { Oeste } \\
\text { Maranhense }\end{array}$ & 01 & 02 & 04 & 02 & 01 & 01 & -- & 02 & -- & 04 & 06 & 11 \\
\hline Itinga do $M A$ & 01 & 01 & -- & -- & -- & -- & -- & -- & -- & -- & 01 & 01 \\
\hline Lago da Pedra & -- & -- & -- & -- & -- & -- & -- & -- & -- & 04 & -- & 04 \\
\hline Santa Luzia & -- & -- & -- & -- & -- & -- & -- & 02 & -- & -- & -- & 02 \\
\hline Turiaçu & -- & 01 & 04 & 02 & -- & -- & -- & -- & -- & -- & 04 & 03 \\
\hline Zé Doca & -- & -- & -- & -- & 01 & 01 & -- & -- & -- & -- & 01 & 01 \\
\hline $\begin{array}{c}\text { Sul } \\
\text { Maranhense }\end{array}$ & - & -- & -- & -- & -- & -- & -- & -- & 01 & -- & 01 & -- \\
\hline Porto Franco & -- & -- & - & -- & -- & -- & -- & -- & 01 & -- & 01 & -- \\
\hline
\end{tabular}

Fonte: Pesquisa Direta

Tabela 2. Distribuição dos casos de Doença de Chagas Aguda no Estado do Maranhão e registrados no banco de dados do SINAN e da FUNASA entre os anos de 2007 e 2011, de acordo com a mesorregião e municípios de origem.

corresponde a indivíduos com idade entre 19 e 59 anos, sendo 14 casos (51,85\%) no SINAN e 23 casos $(62,16 \%)$ na FUNASA. Quanto à residência, foi verificado que o maior número de casos é proveniente da zona rural, apesar de ainda serem observadas diferenças entre os dois bancos de dados. Enquanto no SINAN o número de pacientes provenientes da zona rural correspondia a 88,89\% dos casos (24 casos), na FUNASA esse valor caiu para $75,67 \%$ (28 casos).

Interessante notar que quando demonstrada a forma de transmissão mais prevalente no estudo, foi verificada uma inversão do perfil quando comparados os dois bancos de dados (Tabela 4).

Enquanto os casos reportados ao SINAN, na sua maioria, foram decorrentes de transmissão vetorial, perfazendo $51,8 \%$ dos casos e sendo $48,1 \%$ por transmissão oral, na FUNASA a maior incidência era por transmissão oral $(62,2 \%)$. Em relação à evolução dos casos, os dois sistemas comportaram-se de forma semelhante (Tabela 4). Enquanto o SINAN reportou 2 óbitos (7, 4\%) durante o período de estudo, na FUNASA foi notificado apenas $1(2,7 \%)$. 


\begin{tabular}{|c|c|c|c|c|}
\hline & \multicolumn{2}{|c|}{ SINAN } & \multicolumn{2}{|c|}{ FUNASA } \\
\hline & $\mathbf{N}$ & $\%$ & $\mathbf{N}$ & $\%$ \\
\hline \multicolumn{5}{|l|}{ Sexo } \\
\hline Masculino & 16 & 59,26 & 23 & $62,16 \%$ \\
\hline Feminino & 11 & 40,74 & 14 & $37,84 \%$ \\
\hline \multicolumn{5}{|l|}{ Faixa Etária } \\
\hline 0-11 ANOS & 08 & 29,63 & 05 & $13,51 \%$ \\
\hline $12-18$ & 01 & 3,70 & 04 & $10,81 \%$ \\
\hline $19-59$ & 14 & 51,85 & 23 & $62,16 \%$ \\
\hline$\geq 60$ ANOS & 04 & 14,81 & 05 & $13,51 \%$ \\
\hline \multicolumn{5}{|l|}{ Moradia } \\
\hline Urbana & 03 & 11,11 & 09 & $24,32 \%$ \\
\hline Rural & 24 & 88,89 & 28 & $75,68 \%$ \\
\hline
\end{tabular}

Tabela 3. Distribuição dos casos de Doença de Chagas Aguda no Estado do Maranhão e registrados no banco de dados do SINAN e da FUNASA entre os anos de 2007 e 2011, de acordo com o sexo, faixa etária e moradia.

\begin{tabular}{lcclcc}
\hline & \multicolumn{2}{c}{ SINAN } & & \multicolumn{2}{c}{ FUNASA } \\
\cline { 2 - 3 } \cline { 5 - 6 } & $\mathbf{N}$ & $\mathbf{0}$ & & $\mathbf{N}$ & $\mathbf{\%}$ \\
\hline Forma de & & & & & \\
Transmissão & & & & & \\
Vetorial & 14 & 51,85 & & 14 & 37,84 \\
Oral & 13 & 48,15 & & 23 & 62,16 \\
Acidental & -- & -- & & -- & -- \\
Outra & -- & -- & & -- & -- \\
$\quad$ Evolução do & & & & & \\
$\quad$ Caso & & & & & \\
Vivo & 25 & 92,59 & & 36 & 97,30 \\
Óbito & 2 & 7,41 & & 01 & 2,70 \\
Fonte: Pesquisa Direta & & & & &
\end{tabular}

Tabela 4. Distribuição dos casos de Doença de Chagas Aguda no Estado do Maranhão e registrados no banco de dados do SINAN e da FUNASA entre os anos de 2007 e 2011, de acordo com a forma de transmissão e a evolução do caso.

\section{Diselssão}

Este trabalho faz uma comparação entre as informações colhidas pelo SINAN e a FUNASA quanto à vigilância epidemiológica da Doença de Chagas Aguda no estado do Maranhão. Nossos resultados mostram, pela primeira vez, que existem divergências entre os dois sistemas de registros, em relação a todos os parâmetros avaliados para DCA.

$\mathrm{O}$ estudo mostrou um menor número de registros de casos de DCA no SINAN em relação à FUNASA (37\%). O menor número de casos registrados no SINAN pode indicar uma subnotificação da doença por esse sistema. Porém quando analisados o número de notificações por ano observa-se um perfil diferente. Em 2008 e 2009 o maior número de casos foi reportado no SINAN, indicando que o problema não é apenas de subnotificação.

É sabido que somente os casos de Doença de Chagas Aguda são de notificação compulsória ${ }^{10}$. Logo, nesta situação, é possível que casos de Doença de Chagas Crônica tenham sido notificados como forma aguda da doença, levando ao erro de preenchimento. Fatores como a falta de experiência dos técnicos responsáveis pelo abastecimento do banco de dados podem estar relacionados a essas situações.

As divergências de notificação podem estar relacionadas, também, às diferenças quanto ao abastecimento dos dois bancos de dados. Enquanto os dados fornecidos ao SINAN provêm das unidades locais de saúde espalhadas por todo o estado, na FUNASA a vigilância epidemiológica, confirmação dos casos e abastecimento do banco de dados são centralizados na sede regional. Quando feita a notificação de alguma suspeita de caso de Doença de Chagas um técnico da fundação é deslocado para o local de ocorrência para que seja feita a confirmação dos casos.

Essa hipótese é confirmada quando se observa que, também, não existe congruência quanto ao município de origem dos casos confirmado da doença, em relação aos dois bancos de dados. Enquanto foram reportados à FUNASA casos da forma aguda da doença nos municípios de São João do Sóter, Presidente Sarney, Rosário, São Luis, Lago da Pedra e Santa Luzia, os mesmo não foram observados no SINAN. Por outro lado a FUNASA não notificou alguns casos apontados pelo SINAN como sendo municípios afetados pela doença.

Segundo a FUNASA a mesorregião mais atingida foi o Norte Maranhense (16 casos), seguida pelo Oeste Maranhense (11 casos). Contudo percebe-se que há maior periodicidade no Oeste Maranhense, visto que foi notificado pelo menos um caso em cada um dos anos de estudo. Isso pode ser devido à proximidade da região amazônica, onde se verifica um maior indicie da doença em relação ao país'11. Essas diferenças entre os dois bancos de dados levaram, também, a divergências quanto ao perfil dos pacientes e da doença.

Exceto quanto à forma de transmissão da 
doença, todas as demais variáveis seguiram o mesmo padrão de comportamento. Nesse caso, o SINAN reportou a transmissão vetorial como a mais prevalente, ao passo que a oral foi a mais citada segundo os dados da FUNASA. Novamente, os dados da FUNASA parecem ser mais confiáveis visto que,trabalhos têm mostrado que nos últimos anos ocorreu uma redução da transmissão vetorial, com aumento da transmissão pela via oral12.

A faixa etária mais atingida, segundo a FUNASA, foi a adulta (19 a 59 anos), representando $62,1 \%$ dos casos. Esse mesmo perfil também foi verificado em outros estudos ${ }^{13}$.

Em relação ao sexo, a doença atingiu, principalmente, indivíduos do sexo masculino. Esse maior percentual de casos da doença nos homens também foi mostrado por Cutrim et $\mathrm{al}^{7}$, quando avaliou o perfil da doença no estado do Maranhão, porém em um período diferente, e por Bozelli et al ${ }^{14}$ que estudou a doença no estado do Paraná.

Também foi demonstrado que há uma predominância da doença de Chagas em regiões rurais. Isso é reflexo, principalmente, das condições de moradia. Geralmente esses indivíduos moram em casas de "pau-a-pique" e cobertas de palha, o que favorece a presença do vetor no domicílio, tornando mais provável a exposição à doença ${ }^{15}$. Por outro lado, segundo a FUNASA, a principal forma de transmissão nesse período foi a oral $(\mathrm{N}=23 / 62,16 \%)$. No entanto, $95,6 \%$ dessas infecções por transmissão oral ocorreram em 2011, devido a um surto da doença ${ }^{16}$.

Geralmente a infecção é adquirida ao ingerir alimentos como açaí e caldo de cana, contaminados com as fezes do inseto vetor infectado ${ }^{17}$. A transmissão oral ocorre em alguns surtos episódicos e já está sendo estudada para melhor entender a sobrevivência e infectividade por Trypanosoma cruzi em alimentos como a polpa de açaí ${ }^{18}$.

Os dados do SINAN e da FUNASA mostraram que, a grande maioria das pessoas sobrevive à Doença de Chagas Aguda, sendo que os valores se aproximaram ( $\mathrm{N}=25 / 92,59$ e $\mathrm{N}=36 / 97,26 \%$ respectivamente). No entanto, a grande maioria dos pacientes obteve cura, pois mesmo que a resposta não tenha sido imediata ao inicio do tratamento, a possibilidade de cura é prevista a médio ou longo prazo $^{14}$.

Dessa forma, é possível verificar que os dados fornecidos pela FUNASA apresentam maior confiabilidade, na medida em que existe um trabalho de campo assíduo e acompanhamento dos pacientes. Contudo, é necessário que os dados dos dois órgãos sejam analisados com mais rigor e que exista uma interação entre eles para que possíveis divergências de informações não ocorram.

\section{Conelusões}

O trabalho apontou alguns problemas em relação à vigilância epidemiológica da Doença de Chagas Aguda no Estado do Maranhão. O SINAN, sistema responsável pela notificação compulsória de diversas doenças de interesse para a saúde pública, apresentou resultados bastante diferentes dos obtidos dos registros da FUNASA, que foi considerada mais confiável. A falta de treinamento dos técnicos responsável pela coleta e abastecimento dos dados e até mesmo o diagnóstico correto são fatores que podem estar envolvidos nos erros de notificação. A notificação correta é uma relevante ferramenta para que se possa combater a doença em sua fase aguda, buscando sua eliminação. Logo, é de fundamental importância o estabelecimento de mecanismos que busquem melhorar a vigilância epidemiológica e obtenção de dados mais confiáveis.

\section{Agradecimentos}

Agradecemos ao senhor Afonso Carlos Saraiva Luz, Técnico de Sistema do SINAN-SES-MA e ao senhor Irandy Augusto Almeida Técnico da FUNASA pelo auxilio durante a coleta de dados.

\section{Referências}

1. Hotez PJ, Dumonteil E, Woc-Colburn L, Serpa JA, Bezek S, Edwards MS, et al. Chagas disease: "the new HIV/AIDS of the Americas". PLoS Negl Trop Dis. 2012; 6(5):e1498.

2. Lunarderlli A, Borges FP, Mello KF, Zeferino ASA. Soroprevalência da doença de Chagas em candidatos a doadores de sangue. Rev Bras Anal Clin. 2007; 30(2):139-41.

3. Tartarotti E, Azeredo-Oliveira MTV, Ceron CR. Problemática vetorial da Doença de Chagas. Arq Ciênc Saúde 2004; 11(1):44-7.

4. Congenital Transmission of Chagas Disease.Centers for Disease Control and Prevention. 2012; 61(26):4789.

5. Bua J, Volta BJ, Velazquez EB, Ruiz AM, Rissio AMD, Cardoni RL. Vertical transmission of Trypanosoma cruzi infection: quantification of parasite burden in mothers and their children by parasite DNA amplification. Trans Roy Soc Trop Med Hyg 2012; 106:623-8

6. Camargo M, Silva G, Castilho E, Silveira A. Inquérito sorológico da prevalência da infecção chagásica no 
Brasil, 1975 a 1980. Rev Inst Med Trop São Paulo 1984; 26:192.

7. Cutrim FSRF, Almeida IA, Gonçalves EGR, Silva AR Doença de Chagas no Estado do Maranhão, Brasil: registro de casos agudos no período de 1994 a 2008. Rev Soc Bras Med Trop 2010; 43(6):705-710.

8. Portal da Saúde [homepage onthe Internet]. Brasília: $\mathrm{O}$ que é o sinan. Disponível em: <http://dtr2004.saude.gov.br/sinanweb/ > data de acesso: 14.12.2012.

9. Divisão do Brasil em mesorregiões e microrregiões/ Fundação Instituto Brasileiro de Geografia e Estatística, Departamento de Geografia - Rio de Janeiro: IBGE, volume 2 - Região Nordeste, 1990.

10. Serra, J. Lista Nacional de Doenças de Notificação Compulsória. Inf. Epidemiol. Sus [online] 2000; 9(1):59-60.

11. Coura JR, Barrett TV, Arboleda MN. Ataque de populações humanas por triatomíneos silvestres no Amazonas: uma nova forma de transmissão da infecção chagásica? Rev Soc Bras Med Trop 1994; 27:251-253.

12. Brasil. Ministério da Saúde. Secretaria de Vigilância em Saúde. Guia de vigilância epidemiológica / Ministério da Saúde, Secretaria de Vigilância em Saúde. - 6. ed. Brasília : Ministério da Saúde, 2005. 816.

13. Camargo M, Silva G, Castilho E, Silveira A. Inquérito sorológico da prevalência da infecção chagásica no Brasil, 1975 a 1980. Rev Inst Med Trop São Paulo 1984; 26:192.
14. Bozelli, CE, Araujo SM, Guilherme ALF, Gomes ML. Perfil clínico-epidemiológico de pacientes com doença de Chagas no Hospital Universitário de Maringá, Paraná, Brasil. Cad. Saúde Pública 2006; 22(5):1027-1034.

15. Monteiro MW, Barbosa MGV, Toledo MJO,Fé FA, Fé NF. Série de casos agudos de doença de Chagas atendidos num serviço terciário de Manaus, Estado do Amazonas, de 1980 a 2006. Rev Soc Bras Med Trop 2010; 43(2):207-210.

16. Doença de Chagas preocupa autoridades sanitárias. Jornal O Estado do Maranhão 201120 out. Avaliable from:http://imirante.globo.com/noticias/2011/10/2 $0 /$ pagina288688.shtml.

17. Carvalho, JLS. Importância das espécies vetores da doença de Chagas no Município de Posse, Estado de Goiás, com especial referência a Triatomasordida, na manutenção da transmissão endêmica de Trypanosoma cruzi [Dissertação]. Brasília: Universidade de Brasília; 2009.

18. Passos LAC, Guaraldo AMA, Barbosa RL, DIAS VL, Pereira KS, Schmidt FL et al. Sobrevivência e infectividade do Trypanosoma cruzi na polpa de açaí: estudo in vitro e in vivo. Epidemiol. Serv. Saúde. 2012; 21(2):223-232. 